\title{
Report of the Second IMMLEP Task Force Meeting 1-5 December, 1975
}

\begin{abstract}
Leprosy workers evervwhere are aware of the great importance of the World Health Organisation Special Programme for Research and Training in Tropical Diseases, with the establishment of IMMLEP, the Task lorce dedicated to the discovery of a specific vaccine against Mycobacterium leprae. A summary report of the first meeting of IMMLEP was reprinted with the permission of the W'orld IHealth Organisation in Leprosy Review, Vol. 47, No. 3. We are happy to print here, also with permission, the Report of the Second IMMLEP Task Force Meeting.
\end{abstract}

Three specific goals were established for the IMMLEP Task Force at its initial meeting in November 1974. They were briefly: (a) To develop M. leprae specific skin test antigens suitable for carrying out epidemiological studies of leprosy; (b) to develop a vaccine for leprosy which would induce sensitization for cell-mediated immunity to $M$. leprae and engender resistance to the disease, and (c) to understand the nature of the pathological complications of leprosy so that they can be prevented or better treated. While the great hope of the programme is to be able to immunize against leprosy, the Task Force remains aware that the induction of sensitivity to antigens of $M$. leprae does not lead automatically to production of protective immunity. Only with appropriate testing and clinical assessment of protective effect, will it be possible to know if the vaccine can be developed.

The present second IMMLEP Task Force meeting, held December 1975, has as its focus the epidemiological problems of leprosy. A summary of the achievement of one year of research by the Task Force members present included: (a) An improved method of purification of bacilli containing less than $1 \%$ of contaminating material from armadillo tissue; (b) development of three different soluble skin test antigen preparations, all of which demonstrated in preliminary trials considerable $M$. leprae skin-test reactivity in tuberculoid leprosy patients and low levels of skin-test reactivity with lepromatous or non-exposed individuals; (c) three small-scale field studies were carried out on two of the soluble antigens, and one large epidemiological survey (2300 tests) was carried out with one of the products; (d) a mycobacterial infection in some wild armadillos in one district was discovered and the organism involved, in preliminary tests, showed several similarities to $M$. leprae; (e) immunochemical analysis of isolated mycobacteria demonstrated 45 definable components and indicated both that a standardized reference system for antigens of $M$. leprae is feasible, and that individual antigenic components could be purified and monospecific antisera to them produced, and (f) comparison of antigenic properties, both by serological techniques and skin testing, indicated a group of readily cultivated fast growing species with striking antigenic similarities to M. leprae.

On the basis of these findings protocols were prepared for: (a) Calibration and comparison of the available skin test antigens in terms of dose response, and (b) frequency of reactivity in leprosy patients, contacts and healthy individuals in non-endemic areas. First formulations were prepared of protocols for testing three epidemiological hypotheses relating skin test reactivity to: (a) Susceptibility to 
disease; (b) exposure to bacilliferous leprosy patients; (c) subclinical infection; (d) exposure to environmental mycobacteria, and (e) responsiveness to a future vaccine when developed, and the order of priorities suggested. Ethical considerations are a permanent feature of all IMMLEP studies.

In pursuit of the specific goals of IMMLEP, future plans include: (a) Continued follow-up of skin-lest antigen purification and testing, (b) analysis of the epidemiological data obtained so far, and (c) initiation of vaccination studies including optimal use of adjuvants in laboratory animals, with progress in those areas to be reviewed at a third IMMLEP Task Forice meeting to he held in a year's time.

\section{List of Participants}

I) R. BERGQUIST, Armauer Hansen Research Institute, Addis Ababa, Ethopia.

Dr B. R. BLOOM, Albert Finstein College of Medicine, Bronx, New York 10461, United States of America.

Dr J. CONVIT, Instituto Nacional de Dermatologia, Caracas 101, Venezuela.

Dr P. DRAPER, National Institute for Medical Research, Mill Hill, London NW7 I AA, United Kingdom.

Dr T. GODAL, The Radium Hospital, Montabello, Oslo 3, Norway.

Dr M. HARBOE, Institute of Experimental Medical Research, University of Oslo, Ullevaal Iospital, Oslo 1, Norway.

Dr W. F. KIRCIHEIMER, Laboratory Research Branch, U.S. Public Health Service Hospital, Carville, Luisiana 70721, United States of America.

Dr B. D. MOLESWORTH, LEPRA Control Project, Blantyre, Malawi.

Dr S. K. NOORDEN, Central Leprosy Teaching \& Research Institute, Chingleput, Tamil Nadu, India.

Dr R. J. W. REES, National Institute for Medical Research, Mill Hill, London NW7 I AA, United Kingdom.

Dr C. C. SHEPARD, Leprosy \& Rickettsia Branch, Virology Division, Center for Disease Control, At lanta, Georgia 30333, United States of America.

Dr M. J. SHIELD, School of Pathology, Middlesex Hospital Medical School, London W1 P 7LD, United Kingdom.

Dr J. L. STANFORD, School of Pathology, Middlesex Hospital Medical School, London W1 P 7LD, United Kingdom.

Dr G. P. WALSH, Gulf South Research Institute, New Iberia, Louisiana 70560, United States of America.

\section{SECRET ARIAT}

Dr H. SANSARRICQ, Chief, Leprosy, WHO, Geneva, Switzerland.

Dr J. WALTER, Medical Of ficer, Leprosy, WHO, Geneva, Switzerland.

Dr M. L. BRUBAKER, Regional Adviser, Leprosy and Venereal Diseases, AMRO, Washington, United States of America.

Dr G. TORRIGIANI, Acting Chief, Immunology, WHO, Geneva, Switzerland.

Dr E. DE MAAR, Special Programme for Research and Training in Tropical Diseases, WHO, Geneva, Switzerland.

Dr K. L. HITZE, Chief (alternate: Dr J. Guid), Tuberculosis, WHO, Geneva, Switzerland.

Mr T. SUNDARESAN, Health Statistical Methodology, WHO, Geneva, Switzerland.

\section{Summary Report}

\section{INTRODUCTION}

At the direction of the Steering Committee, the second Task Force of IMMLEP met from 1 to 5 December, 1975, in Geneva to consider the results obtained so far, and to formulate further investigations of the new skin test reagents related to leprosy. The Task Force comprised the 
members of the Steering Committee, 5 members of the 1974 Task Force meeting, 5 new members, and 5 members of the WHIO secretariat.

The present Task Force activities related to the development of the new skin test reagent's may be summarized as follows.

\section{STATUS OF $M$. LEPRAE-DERIVED SKIN TEST ANTIGENS}

Three kinds of skin test reagents have been prepared from leprosy bacilli by Task Force members, and two of them have been subjected to preliminary field testing.

\section{Skin test reagent $L C$}

This reagent was elaborated by Dr J. Convit from supernatants of lepromins prepared from heavily infected human and armadillo tissues. (Lepromins are suspensions of whole killed leprosy bacilli, crudely separated from tissue.) Whole lepromins evoke 2 types of reaction in sensitized persons. Firstly, the Fernandez reaction producing an ery thematous phase, oedema and induration $48 \mathrm{~h}$ after injection, and in some ways analogous to the tuberculin reaction. Secondly, the Mitsuda reaction, producing a tuberculoid granuloma at the site of injection 3 weeks to 1 month later. This latter reaction is a response to whole bacilli in the reagent, and the former reaction is to soluble antigens. Thus, Dr Convit's reagent (LC) is designed to evoke the Fernande $z$ and not the Mitsuda reaction.

Results (Bull. Wld Hlth Org. (1975), 52, 193-197) were presented of skin tests carried out with the entire supernatant and with ammonium sulphate precipitates of this material, reconstituted to the original volume. These reagents produced equivalent results and their activity was removable by trypsinization. Using the reagents, small groups of patients with lepromatous and tuberculoid disease were found negative and positive respectively as defined in Dr Convit's publications. Villagers in 3 villages, 1 in Chile where leprosy is not known to occur, and 2 in Venezuela with different levels of leprosy prevalence, were tested with these reagents, with whole lepromin, and with the tuberculin PPD. There were less than $4 \%$ of persons producing positive reactions in the Chilean village, and $45 \%$ of adults were positive in the Venezuelan villages. However, in the Venezuelan village of lowest endemicity, $29 \%$ of children were positive, and in the village of greatest endemicity the percentage of positive children was $45 \%$ and did not differ from that of the adults.

\section{Skin test reagent $L R$}

This is prepared by Dr Rees and Dr Draper from leprosy bacilli killed by irradiation and extracted from infected armadillo tissues. The bacilli are exhaustively freed of tissue antigen by methods outlined in Protocol No. 2/75 of this summary, and then broken by ultrasound. The solution is freed from particles by centrifugation and filtration through membrane filters with a pore size of $0.22 \mu \mathrm{m}$. This reagent is diluted for use according to protein concentration. Several batches have been prepared and tested in 6 countries.

The earliest batch, LRA4, was tested firstly in Malaysia and Ethopia on very small numbers of well documented lepromatous and tuberculoid leprosy patients. From these tests a dose of $2 \mu \mathrm{g}$ protein in $0.1 \mathrm{ml}$ was selected for testing on secondary schoolchildren and tuberculosis patients in Libya. One hundred and fifty persons were tested in Libya and the reagent was found to be too concentrated. In view of this, a reaction size of $12 \mathrm{~mm}$ was selected as positive, and by this criterion $8 \%$ of the schoolchildren and $16 \%$ of the adult tuberculosis patients produced positive reactions. (Leprosy is considered to have a prevalence of 3/1000 in Libya.) An improved batch, LRA6, was tested on volunteer university students in England and Norway where leprosy is non-endemic. Two doses, $2 \mu \mathrm{g}$ and $0.2 \mu \mathrm{g}$ of protein of the reagent were tested simultaneously with tuberculin. One out of 70 Oxford students and 11 out of 60 Oslo students reacted positively to LRA6. There was a relationship between LRA6 positively and strong positivity to 5 TU of PPD (RT23) in the Norwegian data of Dr Harboe. In other areas (Oxford and Libya) such a relationship has not been found. The exploration of this apparent inconsistency is unknown and the specificity of LRA6 will be further investigated in non-endemic areas for leprosy (see Protocol No. 5/75). 
LRA6 was assessed in 1500 persons as part of a larger study in Burma, using a dose of $0.2 \mu \mathrm{g}$ protein. It was found that $3 \%$ of patients with lepromatous leprosy and $39 \%$ of those with tuberculoid leprosy produced positive reactions as compared with $18 \%$ among the general adult population. An interesting sex difference was noted in the general population, amongst tuberculoid patients and amongst contacts of lepromatous disease; in each case, women were markedly less reactive than men, and this was especially so amongst the close contacts. The findings amongst villagers from villages with differing prevalences of leprosy were interesting. It was found that reactivity to LRA6 reached a peak at a prevalence of $3 \%$ where up to $70 \%$ of persons from some villages produced positive reactions. At higher prevalences the percentage of positive reactors was $25 \%(+$ or $-10 \%$ ). (The nature of this phenomenon will be further pursued as outlined in Protocols Nos. 7-8/75.)

Skin test reagents prepared by Dr Stanford and Dr Shield from 21 other species, subspecies or serotypes of mycobacteria were also used in the Burma study, and 13 of them were compared with LRA6 (see working paper; Annex 1).

As found previously a relationship between certain of these and $M$. leprae was detected. Three reagents in particular (vaccin B, nonchromogenicin and lactin) were found to produce results similar in many ways to those obtained with LRA6. The remaining reagents fell into two groups, those with some relationship to the leprosy bacilli (chelonin, vaccin, vaccin $A$, diernhoferin, smegmatin and gordonin), and those apparently unrelated (flavescin, xenopin and marianin); one reagent (marinin) was considered unreliable. Many results of the study carried out in Burma still await assessment.

\section{Skin test reagent $L K$}

This reagent was prepared by Dr Kirchheimer (Leprosy in India (1975), 47, No. 3, 142-150) in small quantities from leprosy bacilli carefully separated from frozen armadillo livers. The organisms were broken by mechanical means and by ultrasound, and the soluble part was separated. Protein was precipitated with trichloracetic acid and redissolved in buffer. A small group of lepromatous and tuberculoid leprosy patients were tested with $116 \mu \mathrm{g}$ doses of this material. The very few results available look favourable.

\section{EPIDEMIOLOGICAL STUDIES WITH LYMPHOCYTE TRANSFORMATION TESTS}

Dr Bergquist presented an interim report of the results of the, as yet, incomplete study of LTTs carried out in an area of Ethopia on contacts of lepromatous or tuberculoid leprosy patients and control persons not known to have contact with any form of the disease. The antigens employed were $M$. leprae (whole organisms, a filtered sonicate, and dharmendra antigen), BCG, $M$. avium, and $M$. gordonae (whole organisms). Although some differences were found, the study taken as a whole did not show a significant variation of responses between the 3 groups tested. The results will require further analyses as more information is added to the study.

\section{PREPARATIONS FOR NEW EPIDEMIOLOGICAL STUDIES}

(a) The epidemiology of leprosy in selected areas was reviewed. See working papers Annexes Nos. 2, 3 and 4.

(b) New studies in non-endemic areas. Consideration was given to comparative studies on the presently available skin test antigens (LRA6 and LC) to establish their specificity versus tuberculin. The study will be carried out as set out in Protocol No. 5/75.

(c) The potential application of $M$. leprae derived skin test antigens as outlined at the last Task Force meeting (see IMM/73.3 Protocols Nos. 8 and 9) were reassessed (see Protocols Nos. 7-11/75).

The priority for these studies recommended by the Task Force to the Steering Committee was that the first study to be undertaken should be TDR/IMMLEP/75.8, Protocol No. 5/75. Protocol No. 6/75 will be carried out subsequently to Protocol No. 5/75. This protocol may

* Accepted by the Steering Committee at its meeting on 5-6 December, 1975. 
have to be modified depending on the outcome of Protocol No. 5/75 study. Further epidemiological studies, Protocols Nos. 7, 8, 9/75 should, if possible, be carried out as a joint prospective study after completion of Protocol No. 6/75.

\section{Supply of M. leprae}

Infected armadillo tissue will be submitted to the IMMLEP programme as agreed at the last Task Force meeting (IMM/74.3 Protocol No. 1). However, the yields from sacrificed animals have been lower than that found in those dying from advanced disease (see Protocol No. 2/75, Annex). Thus, the Task Force recommended strongly that the Steering Committee should make plans immediately for expanding the supply of $M$. leprae to meet the needs of the programme in the future.

Mycobacterial infection of wild armadillos from certain areas was reported by one of the centres. Preliminary observations indicate that the mycobacterium in question may be $M$. leprae. A collaborative investigation with public health authorities has been started to define the possible public-health importance of this animal reservoir. The precautions necessary to ensure a non-contaminated supply of $M$. leprae were discussed in detail and set out in Protocol No. $1 / 75$.

\section{PURIFICATION OF M. LEPRAE FROM TISSUES}

An improved system for preparing electron-microscopically clean suspensions of $M$. leprae from infected armadillo tissues was discussed (Protocol No. 2/75). The method resembled in part that of Kirchheimer (see above) and that described previously (IMM/74.3, Protocol No. 2, Appendix 1), but separation in an aqueous two-polymer phase system replaced sucrose density gradients.

The importance of determining the effect of the enzymes used on the bacterial antigens was emphasized, and also the need to measure recoveries of bacteria. The method of $\mathrm{IMM} / 74.3$, Protocol No. 2, had been used successfully in several laboratories. It was agreed that the high-speed supernatant fraction from the preparation described in Protocol No. 2/75 should be examined for presence of antigen of the Convit type. Consistent yields of bacteria from tissues were obtained, but were lower than described in IMM/74.3, Protocol No. 2, probably because the tissue now came from killed armadillos rather than animals dying of leprosy (see Protocol No. 2/75, Annex).

\section{CHARACTERIZATION OF M. LEPRAE ANTIGENS}

Dr Harboe described the potentialities of the crossed immunoelectrophoresis system for study, purification and fractionation of antigens of mycobacteria, and urged the necessity for reference antigen/antibody systems to be developed. The method had been used to study antigens of M. lepraemurium and Mycobdcterium BCG and should now be applied to M. leprae as purified bacteria became available. Reference systems for $M$. leprae antigens based on both precipitation in gel methods and cell mediated immune systems are highly desirable.

\section{GENERAL APPRAISAL}

From the documents presented at the meeting and from the discussions, the progress made since the first Task Force meeting can be summarized as follows.

(1) The supply of infected armadillo tissues has been met as committed in the first Task Force meeting.

(2) A procedure for purification of $M$. leprae has been established and further improved to the extent that the antigenic material produced contained less than $1 \%$ of host tissues.

(3) A bank of armadillo infected tissues has been established and materials distributed to the various investigators.

(4) The methods for an antigen reference system have been established.

(5) Three skin test preparations have been developed.

(6) The results of preliminary field studies undertaken with two available antigens can be summarized as follows:

(a) A supernatant from Mitsuda type lepromin ( $\mathrm{LCH})$ prepared from armadillo or human 
heavily infected tissues (autoclaved preparation) showed a difference in reactivity of populations in endemic and in non-endemic areas.

(b) A protein extract of purified bacilli from armadillo infected tissues (LRA6) showed a high reactivity in polar tuberculoid leprosy patients, and very little reactivity in lepromatous patients. The results obtained in non-endemic areas are at present conflicting.

Extensive studies in a highly endemic area have been carried out and the results are being assessed.

(7) New studies using the LTT have been reported.

(8) Protocols on methods for new studies making use of existing antigenic preparations as well as of others which could be developed, have been designed during this meeting.

(9) Administrative procedures for the Task Force have been established (see Interim Report of ST Chairman, 1975, Annex 5).

The cost involved in these achievements, which only represent a part of the total IMMLEP activities, is only in the order of US\$75,000, all administrative costs for 1975 inclusive.

\section{List of Protocols}

No.

Title

1/75 Supply of $M$. leprae for IMMLEP programme.

2/75 Proposed system for preparing purified suspensions of $M$. leprae from tissues of infected armadillos.

3/75 Purification and characterization of $M$. leprae antigens.

4/75 General considerations for skin test studies to be undertaken in endemic areas.

$5 / 75$ Comparative testing in non-endemic areas.

6/75 Calibration and comparison of available antigens.

$7 / 75$ Possible desensitization as a consequence of heavy exposure to bacilliferous lepromatous patients.

8/75 Relation of skin-test unresponsiveness of contacts to their susceptibility to disease.

9/75 Subclinical infection.

10/75 Environmental mycobacteria.

$11 / 75$ Skin tests in relation to trials of potential vaccines.

\section{PROTOCOL NO. 1/75. SUPPLY OF M. LEPRAE FOR IMMLEP PROGR AMME}

The Task Force reviewed the procedures to be followed in the light of a recent report by $\mathrm{Dr}$ Walsh from the Gulf South Research Institute, that a mycobacterial infection had been found in wild armadillos captured in sout-west Louisiana. While it was agreed that there was insufficient evidence at present to establish beyond doubt that any of these were $M$. leprae infections, most of the available data are not inconsistent with such a conclusion.

The importance and implication of this observation was considered as it related to the IMMLEP programme and commitment. Fortunately, the tissues from the infected wild animals have been distributed rapidly to some IMMLEP members and others. At tempts to identify these mycobacteria are under way. In addition, a detailed sampling programme of wild armadillos in Louisiana and neighbouring states has been undertaken by the Center for Disease Control (United States of America) (60 animals from 10 areas). It is anticipated that more definitive data will be available within 3 to 4 months. This information will be made available to IMMLEP.

In the meantime the following procedures were accepted:

\section{Screening of eight- and nine-banded armadillos prior to inoculation with $\mathrm{M}$. leprae}

(a) Clinical examination. Full clinical examination at the time of capture, and all animals with skin nodules or ulcerations, or enlarged lymph nodes, would be killed. Smears from the above sites would be stained for AFB, and tissues from animals with positive smears will be cultured and subjected to histopathological examination. (From the centres in the United States of America data will be reported to CDC, and the information obtained from all centres is to be reported to IMMLEP.) 
(b) Full hacteriological examinations of clinically normal animals. (1) Ear skin: Scrape or tissue clip to be examined for AFB; ear clip tissue also to be examined histologically. (2) Blood: Examination of buffy coat for presence of AFB. (3) Nasal smear: Examination for AFB.

Cultures for mycobacteria will be set up on all AFB positive material.

All AFB positive animals are to be killed and tissues taken for histopathological examination. CDC and IMMLEP are to be notified as in 1(a). All tests currently available and applicable to the identification of acid-fast organisms as $M$. leprae should be carried out on representative animals on a continuing basis.

2. Quarantine of armadillos before inoculation with $\mathrm{M}$. leprae

Animals that are AFB negative will be held for:

(1) Four months in a holding area isolated from contact with wild armadillos and as protected as possible from rodents and arthropods. They will then be re-examined as in $1(\mathrm{~b})$ just before inoculation (procedure for GSRI and Venezuela); or

(2) For 2 weeks and then they may be inoculated with $M$. leprae as long as ear clips are taken 3 and 6 months later for bacteriological and histological examination (procedure for Carville).

(In future all animals failing to acclimatize to captivity should be killed and autopsied.)

\section{Inoculation with $\mathrm{M}$. leprae and subsequent isolation}

All animals will be inoculated by intravenous route as previously agreed. However, in future only $M$. leprae from leprosy patients or from first passage armadillos currently held at Carville and Venezuela will be used. Until further notice no armadillo passaged $M$. leprae from GSRI will be used for inoculation.

All M. leprae inoculated armadillos will be kept separately from animals awaiting inoculation.

In order to produce heavy infection in a high proportion of animals with $M$. leprae obtained from patients, IMMLEP will provide the centres at GSRI, Carville and Caracas with fresh suspensions containing large numbers of $\mathrm{AFB}$ on wet ice or liquid nitrogen. It is hoped to be able to provide sufficient $M$. leprae for each animal to receive $1.0 \times 10^{8} \mathrm{AFB}$ intravenously.

Various procedures are being explored to predict the optimal time for killing infected animals.

\section{Source of wild armadillos}

Until further information is available from the present studies, only armadillos obtained from areas in which mycobacterial infection has not been found will be used for IMMLEP.

\section{Investigations at time of harvesting from infected armadillos for passage}

From each animal, skin and peripheral nerves will be submitted for histological examination for the presence of AFB in dermal or peripheral nerves.

Fromeach animal a fresh sample of bacilli will be prepared for:

(a) Pyridine test.

(b) (D)-Dopa test.

(c) Fluorescent antibody test (air-dried smears to be prepared on thin glass slides; test will probably be undertaken by Dr Abe).

(d) Mouse foot pad inoculation.

Other tests may be proposed depending on the results from further studies on bacilli from the freshly trapped and mycobacterially infected animals.

\section{Investigations at time of harvesting tissues from armadillos for distribution through IMMLEP.}

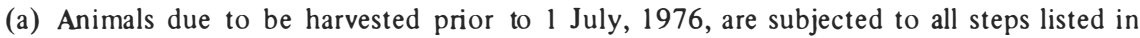
section 5 above.

(b) After this time, animals inoculated with human material or first passage armadillo 
material will be subjected to cultural procedures for mycobacteria, to skin and peripheral nerve histopathology, and whichever of the tests in section 5 are found most specific.

\section{ANNEX TO PROTOCOL NO. 1/75. SUPPLY OF M. LEPRAE 1976-1978}

\section{Supply centres}

The following numbers of heavily infected animals will be available in 1976:

Gulf South Research Institute, New Iberia, Louisiana, United States of America-up to 15 armadillos;

USPHS Hospital, Carville, Louisiana, United States of America-up to 3 armadillos.

The following numbers will be available in 1977:

Gulf South Research Institute-up to 15 armadillos;

USPIIS Ilospital-up to 10 armadillos;

Instituto Nacional de Dermatologia, Caracas, Venezuela-up to 5 armadillos (of the smaller 8-banded species).

The following number will be available in 1978:

Gulf South Research Institute-up to 15 armadillos;

USPHS Hospital-up to 15 armadillos;

Instituto Nacional de Dermatologia-up to 5 animals.

PROTOCOL NO. 2/75. PROPOSED SYSTEM FOR PREPARING PURIFIED SUSPENSIONS OF M. LEPR AE FROM TISSUES OF INFECTED ARMADILLOS

Tissues used so far: liver, spleen, subcutaneous leproma.

All were frozen at $-20^{\circ} \mathrm{C}$ or (usually) at $-70^{\circ} \mathrm{C}$. All were sterilized by $2.5 \mathrm{M} \mathrm{rad}\left[{ }^{60} \mathrm{Co}\right]$ gamma radiation.

(There seems no reason why: (a) Fresh tissues might not be used, or (b) why unirradiated tissue might not be processed. However, the latter alternative involves the hazards of: (i) infection with $M$. leprae of the operator, and (ii) contamination of the digests with other organisms.)

\section{Materials needed}

(1) $\mathrm{SE}$ buffer (0.3 M-sucrose, $0.25 \mathrm{mM}$-dipotassium EDTA adjusted to $\mathrm{pH} 7.1$ to 7.2 with $\mathrm{KOH}$.

(2) Triton $\mathrm{X} 100$ (conveniently handled as $20 \% \mathrm{w} / \mathrm{v}$ solution).

(3) Tris buffer $(0.1 \mathrm{M}$-Tris adjusted to $\mathrm{pH} 7.2$ with $\mathrm{HCl})$.

(4) Tween $(0.05 \%$ Tween 80 in water).

(5) Sodium azide.

(6) Collagenase (from Clostridium histolyticum; we used "type II". from Koch-Light with low proteinase activity; a cruder type might do).

(7) Trypsin (crystalline).

(8) Chymotrypsin (crystalline).

(9) Pronase (type B from Calbiochem; could be replaced by Streptomyces grisius proteinase from other sources).

(10) PEG (polyethylene glycol 6000 from BDH; 130-170 ethylene oxide units per molecule).

(11) Dextran (Dextran T500 from Pharmacia; mol. wt about 500,000).

(12) Phosphate buffer (conveniently $0.5 \mathrm{M}$-potassium phosphate, $\mathrm{pH}$ 6.9).

(13) Sodium chloride (conveniently $1 \mathrm{M}$ - or $2 \mathrm{M}-\mathrm{NaCl}$ ).

(14) Calcium chloride $\left(\mathrm{M}-\mathrm{CaCl}_{2}\right)$.

\section{Apparatus needed}

(1) Homogenizer (Sorvall Omnimix has especially fast motor and is easy to keep cool; others would probably do).

(2) Centrifuge (capable of spinning samples at $10,000 \times \mathrm{g}$; Sorvall RC-2, RC-2B, RC-5 suitable). 
(3) Separating funnel.

(4) Magnetic stirrer.

llomogenize tissue in SF: buffer, $30 \mathrm{ml}$ buffer/10 $\mathrm{g}$ tissue, $3 \mathrm{~min}$ in Omnimix at top speed, cooled in water with ice.

Centrifuge $500 \times \mathrm{g}, 5 \mathrm{~min}$. Preserve supernatant. Homogenize sediment as before, centrifuge as before, combine supernatants, discard sediment.

Centrifuge 10,000 x g, 10 min. Discard supernatant. Resuspend in SE, centrifuge as before. Pour off supernatant and as much as possible of fluffy top layer of sediment. Keep cold up to this point.

Suspend sediment in $1 \%$ Triton $\times 100$ in SE buffer, stand at room temperature $\left(20^{\circ} \mathrm{C}\right)$ for 1 h to lyse mitochondria.

Centrifuge 10,000 $\times$ g, 10 min, discard supernatant. Wash sediment twice with SE buffer, centrifuging as before. Pour off as much as possible of fluffy upper layer of sediment during washing. There is usually a sharp demarcation between the fluffy uppper layer and the firm lower layer. (There is usually a small whitish (collagen) pellet at the base of the lower layer.)

Suspend in Tris buffer, add a little sodium azide, one drop of calcium chloride, and collagenase at $100 \mu \mathrm{g} / \mathrm{ml}$. Incubate at $37^{\circ} \mathrm{C}, 24 \mathrm{~h}$. (If viahle bacilli were needed, an alternative to axide should be sought.)

Add trypsin and chymotrypsin at $100 \mu \mathrm{g} / \mathrm{ml}$. Incubate $24 \mathrm{~h}, 37^{\circ} \mathrm{C}$. (This step has so far only been necessary with subcutaneous leproma, which gave a suspension with more non-bacterial particles at this stage than did liver.)

Add pronase at $100 \mu \mathrm{g} / \mathrm{ml}$. Incubate $24 \mathrm{~h}, 37^{\circ} \mathrm{C}$. (May be prolonged up to 4 days to cope with large amounts of extraneous protein, but the addition of the trypsin step is probably a more satisfactory alternative.)

Centrifuge $8000 \times \mathrm{g}, 10 \mathrm{~min}$. Wash sediment twice with Tween, centrifuge as before. Suspend in small amount of Tween.

Prepare aqueous two-phase polymer-system. For $100 \mathrm{~g}$ batch use $7.0 \mathrm{~g}$ Dextran, $5.0 \mathrm{~g}$ PEG; water to $75 \mathrm{~g}$ (weigh it!). Stir, or stand and then stir, until polymers are dissolved. (This can conveniently be done by brief stirring after standing overnight.) Add phosphate buffer and sodium chloride to achieve final concentrations of $0.01 \mathrm{M}$ each, add bacterial suspension, add water to $100 \mathrm{~g}$.

Stir system until uniform, then stand in separating funnel until phases separate. Run off lower phase and interface (usually coloured). Dilute top phase with equal volume of water, adding Tween $80(10 \% \mathrm{w} / \mathrm{v}$ solution) to give $0.1 \%$ final concentration. (A $100 \mathrm{~g}$ system gives about $50 \mathrm{ml}$ of upper phase.)

Centrifuge diluted upper phase $8000 \times \mathrm{g}, 30 \mathrm{~min}$. Wash sediment in Tween several times, centrifuging at $8000 \times \mathrm{g}$ for $10 \mathrm{~min}$. (If there is a significant translucent brownish layer above the whitish bacterial layer it may be removed by suspending it gently without disturbing the bacteria.)

Suspend bacteria in water and freeze-dry or preserve as a suspension in Tween $0.05 \%$.

ANNEX TO PROTOCOL NO. 2/75. PROGRESS REPORT ON $M$. LEPRAE AND SKIN TEST ANTIGEN PREPARED FROM INFECTED ARMADILLOS AT THE IMMLEP $M$. LEPRAE BANK (DR REES)

\section{Yield and reproducibility}

Using the definitive system for preparing suspension of $M$. leprae from tissues of infected armadillos developed by P. Draper since the first meeting of the IMMLEP Project Group, held in Geneva in November 1974, highly reproducible yields of purified M. leprae have been obtained. All this data presented in the following table is based on infected tissues from killed armadillos:

* But see Protocol 3/75. 


\begin{tabular}{|c|c|c|c|c|c|}
\hline \multicolumn{3}{|c|}{ Tissue } & \multicolumn{3}{|c|}{$\begin{array}{l}\text { M. leprae } \\
\text { Yield }\end{array}$} \\
\hline $\begin{array}{l}\text { Armadillo } \\
\text { No. }\end{array}$ & Organ & $\begin{array}{l}\text { Wet weight } \\
\text { (g) }\end{array}$ & $\begin{array}{c}\text { Batch } \\
\text { No. }\end{array}$ & $\begin{array}{c}\text { Dry weight } \\
\text { (mg) }\end{array}$ & $\%$ \\
\hline A81 & $\begin{array}{l}\text { Spleen } \\
\text { Liver } \\
\text { Liver }\end{array}$ & $\begin{array}{l}41 \\
40 \\
82\end{array}$ & $\begin{array}{l}\text { A. } 7 \\
\text { A. } 8 \\
\text { A. } 9\end{array}$ & $\begin{array}{c}56 \\
77 \\
166.7\end{array}$ & $\begin{array}{l}0.137 \\
0.193 \\
0.203\end{array}$ \\
\hline A29 & $\begin{array}{l}\text { Liver } \\
\text { Liver }\end{array}$ & $\begin{array}{l}80 \\
80\end{array}$ & $\begin{array}{l}\text { A. } 10 \\
\text { A. } 11\end{array}$ & $\begin{array}{r}102.3 \\
89.5\end{array}$ & $\begin{array}{l}0.128 \\
0.112\end{array}$ \\
\hline
\end{tabular}

Thus, to date $323 \mathrm{~g}$ wet weight infected liver and spleen have yielded $492 \mathrm{mg}$ dry weight $M$. leprae, giving a mean yield of $1.5 \mathrm{mg}$ dry -weight $M$. leprae $/ 1.0 \mathrm{~g}$ wet weight infected tissue. These yields are less than we reported $(2.7 \mathrm{mg} \mathrm{M}$. leprae $/ 1.0 \mathrm{~g}$ tissue) in November 1974 (IMM/74.3, Appendix II, page 12) probably because then our methods were: (1) Less precise and (2) were based on limited information from tissues of infected armadillos which were allowed to progress to death.

To date approximately $300 \mathrm{~g}$ dry weight $M$. leprae have been distributed to the IMMLEP programme.

\section{Purity}

Electron microscopical examination of each of these preparations of bacilli showed them to be free from contamination, including the presence of collagen (see section below on purity of skin test antigen derived from these preparations of $M$. leprae).

\section{Distribution and handling of freeze-dried preparations of $\mathrm{M}$. leprae}

To date, our preparations of purified $M$. leprae have been freeze-dried from water suspensions and like all other purified mycobacterial preparations in water are difficult to resuspend without aggregation. Present studies show that the problem of aggregation can be overcome by initial resuspension in the presence of surfactants and that with these surfactants the maximum concentrations of $M$. leprae in suspension can be obtained under the following conditions:

\section{MAXIMUM CONCENTRATION OF $M$. LEPRAE RETAINED IN SUSPENSIONS FROM FREEZE-DRIED STATE}

Concentration of freeze-dried $M$. leprae $/ \mathrm{ml}$ in suspending fluid
$0.5 \mathrm{mg}$
$0.25 \mathrm{mg}$
$0.1 \%$ Tween $80 /$ saline
$1.0 \mathrm{mg}$
$0.05 \%$ Tween $80 /$ saline
$0.05 \%$ Tween $85 /$ saline

(To obtain a homogeneous suspension the freeze-dried $M$. leprae should be placed in a dry glass homogenizer and the suspending fluid added slowly during grinding.)

From any of the above combinations $M$. leprae will remain homogeneously in suspension at two-fold dilutions adding saline without any further Tween.

\section{SKIN TEST ANTIGEN}

In order to expedite studies on a soluble skin test antigen derived from $M$. leprae obtained from infected armadillo tissues, a large-scale batch of $M$. leprae was prepared from infected armadillo tissues using the earlier method described by P. Draper (Appendix I, IMM/74.3). This preparation has been used throughout for the major skin tests carried out in Burma, Norway and the United Kingdom. There is a stock of 110,600 skin test doses ( $0.2 \mu \mathrm{g}$ protein) of LRA6. 


\section{Reproducihility of antigen}

As agreed by IMMI.FI' small samples of antigen have heen prepared from each new batch of purified $M$. leprae for comparing with LRA6 by skin tests in leprosy patients.

The yield of soluble protein antigen is approximately $25 \%$ of the dry weight of $M$. leprae.

\section{Test for purity}

Quantitative skin tests with protein from uninfected armadillo tissues in groups of guinea pigs sensitized with the same protein or $M$. leprae skin test antigen, incorporated in complete Freund adjuvant, show that LRA6, LRA7 and LRA8 contain $1 \%,<0.68 \%$ and $<0.13 \%$ armadillo protein, respectively.

\section{Standardization of skin test antigen in guinea-pigs}

Pilot studies using animals sensitized with purified preparations of $M$. leprae incorporated in incomplete Freund adjuvant indicate that this method is very sensitive. Preliminary comparative results using I.RA6, LRA7 and LRA8 give similar delayed type hypersensitivity reactions related to their protein content. It is planned to use this method to study cross reactions between $M$. leprae and other species of mycobacteria.

\section{Problems}

(1) The possible effect of four different proteolytic enzymes on antigens of bacteria. Experiments are currently under way using $M$. lepraemurium as a model.

(2) Supernatant from high speed $(10,000 \times \mathrm{g})$ centrifuging probably contains Convit's type of antigen. This should be recovered if suitable techniques become available.

(3) Difficulty of resuspending freeze-dried bacteria.

\section{PROTOCOL NO. 3/75. PURIFICATION AND CHARACTERIZATION OF $M$. LEPRAE} ANTIGENS

\section{Purpose}

The goals are:

(a) To provide a soluble, stable preparation from $M$. leprae which is as specific for M. leprae as possible, cross reacts minimally with other mycobacteria, and is non-sensitizing by skin testing. The product should contain as few immunologically irrelevant, non-reactive components as possible.

The potential uses of this type of $M$. leprae preparation are described in Protocols Nos. 7, 8, 9 and $11 / 75$.

(b) To prepare individual antigenic components from $M$. leprae. Experiments with different preparations of this kind are necessary to obtain information on the relationship between antibody formation and cellular immune reactions against the different components of $M$. leprae in patients with various forms of clinical disease and in individuals exposed to M. leprae who remain healthy.

Sensitive assays for antibody formation against selected $M$. leprae antigens may provide positive findings in infected individuals who develop lepromatous disease with minimal clinical symptoms and who have negative skin tests.

Antibody formation and cell mediated immune reactions against particular antigenic components may be related to important pathological processes such as nerve damage, reversal reactions and ery thema nodosum leprosum (ENL).

Work with purified antigen would facilitate investigations of the taxonomic relationship between $M$. leprae and other mycobacteria.

(c) To establish a reference system for characterization of $M$. leprae by crossed immunoelectrophoresis.

Anti- $M$. leprae antibody should be produced in one laboratory so that antibody and polyvalent $M$. leprae antigen can be sent to other laboratories to serve as a control and reference for their immunodiffusion systems.

Tests with crossed immunoelectrophoresis including direct comparison with the reference 
system are valuable for examination and comparison of various $M$. leprae antigen preparations made for skin testing and other purposes.

(d) The possible use of animal models based on skin tests and in vitro tests of cell mediated immunity for evaluation and comparison of various $M$. leprae antigen preparations should be explored.

(e) To find whether antigens that are important in immune reactions in leprosy have corresponding components with similar structure in readily cultivable mycobacteria.

\section{Materials suitable for fractionation}

(a) Antigens will mainly be isolated from purified leprosy bacilli obtained from infected armadillos.

(b) Some important antigens of $M$. leprae may be present in the tissue fluid of infected armadillo tissue. After the initial homogenization and centrifugation procedures involved in purification of bacilli (see Protocol No. 2/75), the supernatant fluid should be saved and stored at $-20^{\circ} \mathrm{C}$, to be used for antigen fractionation when adequate methods have been developed. Different techniques should be explored for this purpose. Immunoadsorbent procedures based on the use of antisera against $M$. leprae are expected to be particularly useful since they may permit specific recovery of $M$. leprae antigens from the quantitatively dominating animal proteins in the fluid.

(c) Purification of antigens from circulating immune complexes in lepromatous leprosy patients with and without ENL may provide materials that are particularly valuable for establishing the relationship between certain antigenic components of $M$. leprae and clinical course of the disease.

\section{Fractionation procedures}

Development of fractionation procedures using some other mycobacteria is important, to establish the most valuable techniques and to save precious antigenic material, but the work should be applied to $M$. leprae itself as soon as possible.

Several approaches should be explored since it cannot be predicted which methods will lead to purification of antigens most important in protective immunity or clinical complications. Differences in "know how" in different laboratories are also important in this respect.

The separation may be based on conventional biochemical procedures such as salt fractionation, electrophoresis, ion-exchange chromatography, gel filtration, phase partition and lectin-affinity chromatography.

The possibility of using immunological techniques for fractionation should be explored, particularly immunoadsorbent procedures. These are based on the use of antibodies as reagents of high specificity, and make it possible to concentrate the work on fractions that appear to be particularly important from other sources of immunological information. They are particularly suited for work with small amounts of antigen.

Monospecific antisera against individual components of BCG have been made by immunization of animals with precipitates from crossed immunoelectrophoresis, and similar antibodies may be very useful for isolation of individual antigenic components of M. leprae.

\section{Testing of the preparations obtained}

Tests for specificity of antigenic preparation described in section 1(a). The preparation should be characterized by:

(i) Strong reactivity with antisera against M. leprae; (ii) minimal reactivity with antisera against other mycobacteria; (iii) minimal reactivity with antisera prepared against normal armadillo and human tissue; (iv) cell mediated immune reactions as measured by delayed hypersensitivity skin reactions, lymphocyte transformation tests, and migration inhibition tests in animals, and patients with tuberculoid leprosy should show similar specificity and lack of cross reactivity. Skin testing in healthy individuals in leprosy non-endemic areas is described in Protocol No. 5/75.

The stability of the preparation should be ascertained and should include information on the effect of storage at: $-20^{\circ} \mathrm{C}$ and $4^{\circ} \mathrm{C}$, heaping and dilution. 
Tests for purity of preparation described in section l(b):

(a) Precipitable components should give a single band by crossed immunoelectrophoresis or a system of similar sensitivity using a potent, polyvalent anti-M. leprae antibody.

(b) Non-precipitating components with immunological reactivity should be proved homogeneous by adequate biochemical techniques.

PROTOCOL NO. 4/75. (;ENERAL CONSIDERATIONS IOOR SKIN TEST STUDILS TO BE UNIDERTAKEN IN ENDEMIC AREAS

\section{Antigen(s) to be tested}

(1) The antigens to be considered must be as specific as possible for $M$. leprae, stable under the conditions to be tested, and non-sensitizing (cf. Protocols Nos. 7/75 and 3/75).

(2) The optimal dosage for discriminating positive reactors from non-reactors must be known (to be derived from Protocol No. 6/75).

(3) The maximal potency available is required, i.e. a high frequency of reactivity in known tuberculoid leprosy patients (cf. Protocol No. 6/75).

(4) Antigens should be coded.

(5) Additional antigens could be added to this as seems appropriate.

\section{Methodology}

(1) Skin tests should be carried out intradermally in $0.1 \mathrm{ml}$ volume on the volar surface of the forearm.

(2) For multiple tests on single individuals, randomization of test sites is preferable.

(3) Tests must be accurately read by an experienced reader for diameter of induration, diameter of erythema and/or oedema, and subjectively for intensity of erythema (cf. Protocol No. 8/75).

(4) Accurate and detailed records of the study population group must be kept. Some parameters to be included are given in Protocols Nos. 7/75 and 8/75.

\section{Population studied}

In order that the most useful information can be obtained from skin test results, it is important that any population to be tested be characterized with regard to frequency and prevalence of leprosy and tuberculosis, BCG vaccination status, and exposure to environmental mycobacteria. The study group should be group by age, e.g. 6-11, 12-18 and 19-40 years. Sociological parameters which may affect contact with leprosy cases should also be considered (Protocols Nos. 7/75 and 8/75).

In testing populations of leprosy patients, their disease status, determined clinically and histopathologically, should be recorded as follows:

TT, BT, BB, BL, LL and (if possible) I.

Duration of disease, nature of disease (e.g. quiescent, active or reactive), treatment (type, duration and regularity) must be recorded.

\section{Ethical considerations}

(1) It must be appreciated that none of these protocols can be carried out without the approval of the governments concerned, and/or the groups being studied, which will be sought in advance.

(2) All patients with leprosy discovered in the course of any trial should receive treatment.

\section{PROTOCOL NO. 5/75. COMPARATIVE TESTING IN NON-ENDEMIC AREAS}

The purpose is to compare different antigenic preparations from $M$. leprae by skin testing in non-endemic leprosy areas and to delineate whether there is a correlation with tuberculin sensitivity. 
(1) The LRA6 preparation, $2.0 \mu \mathrm{g} / \mathrm{skin}$ test $(0.1 \mathrm{ml}$ intradermally).

(2) The LC preparation as prepared from infected armadillos (LCA) $(0.1 \mathrm{ml}$ intradermally, standardized on the basis of original count of acid-fast bacilli $\left.=1.6 \times 10^{8} / \mathrm{ml}\right)$.

(3) A control preparation made in the same way from lymph nodes from uninfected armadillos standardized to the same total protein concentration.

(4) PPD, RT23, 2 TU/intradermal injection in $0.1 \mathrm{ml}$ volume (obtained from Copenhagen).

Note. Arrangements will be made for the LRA6, LCA and control preparations to be tested for endotoxin (Limulus assay) as a sensitive indicator of the presence of non-M. leprae bacterial products.

\section{Test subjects}

(1) Healthy volunteers, male or female, aged 18-25, who have given informed consent and who have not been exposed previously to any test procedure with $M$. leprae antigens, but who may or may not have been BC.; vaccinated. It should be recorded whether they have been vaccinated once or more, and at which time.

(2) Patients with active pulmonary tuberculosis, sputum positive at time of diagnosis and within 18 months of commencement of treatment. The drug regimen should be recorded and should not include immunosuppressive agents. No adverse effects or more intense skin reactions have been recorded using 1.5 TU RT23 in cases of pulmonary tuberculosis (J. Guld et al., Bull. Wld Hlth Org. (1958) 19,845-951).

For both groups, individuals from leprosy endemic areas must be excluded.

\section{Areas selected}

(1) Chile (southern part). In this area healthy individuals from 2 additional age-groups (children of 10 years or less, and adults 50 years and over) will be studied.

(2) Norway.

(3) United Kingdom.

\section{Reading and recording of skin tests}

All reactions are to be read at 48 and $72 \mathrm{~h}$. The primary recording should be of both the transverse and vertical diameters of induration in $\mathrm{mm}$. Diameters and in tensity of erythema and oedema are also to be recorded. An experienced skin tester will be allocated for each area. It may be possible to arrange for injections and readings to be made by one person for tests in Norway and the United Kingdom. The reader should not have access to the $48 \mathrm{~h}$ results when reading the tests at $72 \mathrm{~h}$.

\section{Distribution and coding of antigens}

The same antigen preparations should be used in the different areas. To this end, the various preparations will be sent to Dr Rees, NIMR, London, whence they will be distributed in coded, identical bottles.

\section{Group size}

For healthy subjects in the 18-25 year age-groups, 60 in all three areas, and 60 in the 2 additional age-groups in Chile.

In each of the 3 areas 20 patients with pulmonary tuberculosis will be tested.

\section{Suggestions for future follow-up studies depending on the results of the above}

(1) Testing with antigen preparations from environmental mycobacteria. The reaction to LRA6 should be compared with the reaction to similarly prepared antigens from mycobacteria selected from those used in the LRA6 skin test trial in Burma. In addition, studies of the in vitro correlates of delayed hypersensitivity may be performed.

\footnotetext{
* Intensity to be recorded as,+++ and +++ (weak, medium and strong).
} 
(2) It is planned to investigate, in Chile, the influence of previous testing on reaction size and the possibility of sensitization with regard to the LRA6 as well as the LCA antigen, and the antigen from uninfected armadillo tissue. With this end in view, information enabling accurate identification will be obtained from all subjects at the time of first testing.

The interval between the first and one or two subsequent tests should not be less than 6 months.

\section{PROTOCOL NO. 6/75. CALIBRATION AND COMPARISON OF AVAILABLE ANTIGENS*}

\section{Purpose}

(a) To determine the optimum dose of presently available skin test antigens in healthy persons in a highly leprosy endemic area and in patients with tuberculoid leprosy.

(b) To compare the reactivity of these antigens, using optimum doses, in patients with leprosy.

\section{Calibration in schoolchildren in (hinglemut District, India}

1.1. In an ongoing programme of tuberculin testing and BCG vaccination, each antigen wilı be given as a second test in a few hundred children, preferably in a secondary school. These children shall not be tested with similar antigens subsequently.

1.2. Preliminary studies will start in the first instance with LRA6 in a dose indicated by the detailed study of data from Burma. Readings will be made after $72 \mathrm{~h}$.

1.3. LRA6 will be received in $1 \mathrm{ml}$ vials $(2 \mu \mathrm{g} / 0.1 \mathrm{ml})$ for further dilution in the field.

1.4. During the first week a starting dose, probably of $0.25 \mu \mathrm{g}$, will be tested. The dosage will be increased from week to week until a maximum permissible dose is reached (producing the strongest acceptable reaction). The maximum dose available from the preparation is $3 \mu \mathrm{g} / 0.1 \mathrm{ml}$. Around the optimum level, 2 doses will be used alternately to establish the dose-response function.

1.5. Similar studies will be made of the LCA antigen and possibly of an "environmental" antigen. For the LCA antigen, readings will be made at $48 \mathrm{~h}$.

\section{Calibration in tuberculoid patients}

Concurrently or subsequently, suitable dose levels will be established in tuberculoid patients not previously tested with lepromin. In selecting the starting dose, the possibility must be taken into account that reactions in these patients may be stronger than in schoolchildren. About 100 patients may be needed, assuming they are willing to receive multiple tests (which would be preferable). For each antigen, a dose will be chosen with suitable right-hand mode, if possible identical with a dose suitable for use in the general population. In particular for LRA6 and LCA antigen, equivalent doses in terms of the right-hand mode should be chosen.

\section{Comparison of antigens in patients}

3.1. A comparative study in leprosy patients will include LRA6 and LCA antigen, each in a dose thus chosen. RT23, 2 TU is given as a third test, and an "environmental" antigen expected to be antigenically close to $M$. leprae might be given as a fourth test.

3.2. The tests are allocated randomly to skin sites in the forearms in each individual.

3.3. For the duration of the study, all patients will be tested except for authentic tuberculoid cases, identified by clinical examination. A random third of this latter group will be selected for testing. A total of 200 patients tested is probably sufficient.

3.4. The reading should be done by a technician with no knowledge of leprosy immunology. All reactions will be read both after 48 and $72 \mathrm{~h}$.

3.5. Independently of the skin reactions (before the testing) each case will be described classified clinically. For all patients not excluded from testing, material will be collected for histological examination (preferably in duplicate, in India and abroad).

* This protocol may have to be modified dependent on the results of the study outlined in Protocol No. 5/75. 


\section{Replication outside India}

The last study (the comparison in patients) should preferably be carried out according to the same protocol if taken up outside India. Preliminary calibration studies need not be identical and might be limited or omitted if the studies in India are thought sufficient.

\section{PROTOCOL NO. 7/75. POSSIBLE DESENSITIZATION AS A CONSEQUENCE OF HEAVY EXPOSURE TO BACILLIFEROUS LFPROMATOUS PATIENTS}

\section{Hypothesis to be tested}

Is the frequency and intensity of skin test reactions diminished in contacts of active untreated lepromatous patients as compared with contacts of tuberculoid patients and treated (one year) lepromatous patients?

\section{Methodology}

(1) Skin test with a selected antigen.

(2) Appropriate tests of cell mediated immunity if possible, on a sampling basis.

\section{Selection of appropriate area for study}

Requirements. The study should be carried out in a context in which known and predictable household contact with the patient population can most clearly be defined.

Parameters to be considered include: (a) Prevalence and incidence of disease in the area; (b) age; (c) duration of contact; (d) sex; (e) relationship to patient; (f) social habits of population: these must be assessed to establish that the contact of different sexes, age-groups, etc., with lepromatous cases is the same. If there is a difference, it is necessary to define exactly what this difference in degree of contact may be; $(\mathrm{g})$ duration and regularity of treatment of patients; (h) bacteriological examination of nasal smears is to be carried out.

The study should be carried out in an area where the majority of existing cases are known and registered, and can be appropriately classified according to the stage of their disease. The clinical status of the contacts should be established at the beginning and at the conclusion of the study. The population must be relatively stable.

\section{Requirements for the antigen preparation to be used in such a study}

(1) It must show a high frequency of positive reactivity in tuberculoid (TT) leprosy patients (preferably $>80 \%$ ).

(2) It should give low reactivity in lepromatous ( $L L)$ patients $(<5 \%)$.

(3) It should give low reactivity in non-exposed healthy individuals $(<10 \%)$.

(4) The antigen must be non-sensitizing so that changes in reactivity of the control population can be examined at later times.

(5) Appropriate dosages of the antigen, stability and storage conditions must be established.

\section{Anticipated problems}

(1) Contacts may, in fact, have been leprosy patients in the past or may be incubating the disease.

(2) The higher the endemicity of the area, the less reliable will be the predictability of the exposure and non-exposure of the test group to active lepromatous patients. The incidence in the villages selected should not be so high that a plausible low contact control group cannot be identified.

(3) The lower the endemicity, the more difficulty is experienced in finding the required number of contacts.

(4) That of obtaining precise data on the amount and duration of contact.

\section{PROTOCOL NO. 8/75. RELATION OF SKIN TEST UNRESPONSIVENESS OF CONTACTS} TO THEIR SUSCEPTABILITY TO DISEASE

\section{Hypothesis to be tested}

Is consistent failure of contacts to react immunologically to skin tests with M. leprae 
antigens related to:

(a) Increased frequency of clinical disease.

(b) Increased frequency of acquiring the lepromatous form?

\section{Methodology}

(1) Skin tests will be made with antigen meeting requirements specified in Protocol No. $7 / 75$.

(2) A large population will be tested twice with an interval of 2 years. The population group to be studied will consist of the contracts negative to both of the tests. (The group consisting of those who were negative at the first testing but positive at the second one might form part of the population studied according to Protocol No.9/75.)

(3) The susceptibility to disease of these individuals can be evaluated in 2 ways: (a) Prospectively, i.e. the entire study group would be examined for disease 5 years after the second negative test reading. (More frequent examination is desirable if practicable.) (b) Case control method, i.e. cases of leprosy reporting to health services in the indexed study group would be recorded and linked to original skin test status.

\section{Selection of area}

(1) It should be hyperendemic for leprosy.

(2) Careful follow-up must be possible.

(3) The population must be relatively stable.

(4) Since the Burma trial area has high endemicity, and 1500 people there have already received one test with LRA6, it should be considered for such a study, subject to government agreement. A proportion of the general population who subsequently develop leprosy may be subjected to diagnostic lepromin testing in conjunction with the format of the WHO Leprosy BCG trial. In the context of this protocol, therefore, this problem is unavoidable.

(5) There should be a good record keeping system whereby previous skin test results can be traced.

\section{PROTOCOL NO. 9/75. SUBCLINICAL INFECTION}

\section{Hypothesis one}

Only a fraction of the total population exposed to $M$. leprae in a given endemic area will sooner or later acquire the disease. A further fraction of the exposed population may develop subclinical infection and this may be recognizable by testing with an $M$. leprae skin test reagent.

\section{Methodology}

Total populations in low endemic, moderate endemic and high endemic areas should be skin tested once or more to establish incidence of infection in different situations. This could be done by evaluating a proportion of positive reactors according to different variables such as age, sex, contact state, familial aggregation, etc.

Requirements for antigen preparation to be used in such a study would be the same as in Protocol No. 7/75. If tests were to be carried out on a single occasion a sensitizing skin test antigen could be accepted.

\section{Problems}

3.1 Negative skin test reaction, single or persistent, could indicate either: (i) That there has been no exposure to $M$. leprae, or (ii) that there has been exposure to $M$. leprae but no sensitization to the organism for one of several reasons, i.e. (a) low dose, (b) early stages of infection in those that ultimately develop clinical disease (see Protocol No. 8/75).

3.2. If only one test were undertaken, the study would be inconclusive unless a considerably larger proportion of the population as compared to the proportion of leprosy cases to be expected, were found to respond.

3.3. Such a study could be carried out in a more refined way, as outlined in Protocol No. $8 / 75$. 


\section{Ilypothesis $t w o$}

Individuals in a preclinical stage of the disease, but who are destined to develop lepromatous disease, may already show immunological aberrations such as high levels of antibodies to certain $M$. leprae antigens.

\section{Methodology}

In any prospective epidemological study to be undertaken, one may consider the desirability of collecting serum samples from the population. These samples could be kept frozen until lepromatous patients appear and then compared (see Protocol No. 5/75) with samples from those who developed tuberculoid leprosy or remained free of clinical disease.

\section{Problems}

(1) Blood collection may be resented by the population and interfere with their co-operation.

(2) The cost involved in the collection of a large number of serum samples must be considered before this project is undertaken.

\section{PROTOCOL NO. 10/75. ENVIRONMLNTAL MYCOBACTERIA}

The principal reason for investigation of environmental species of mycobacteria is to find a possible alternative to $M$. leprae for production of a vaccine for leprosy.

There also exists the possibility that delayed hypersensitivity to certain environmental mycobacteria might have an important influence on the epidemiology of leprosy, and affect the outcome of skin test and vaccine trials. Mycobacterial species used in this project will be selected as a result of taxonomic studies relating this organism to M. leprae.

\section{Existing knowledge}

1. Experience in the field and the laboratory indicates a close relationship between the leprosy bacillus and environmental mycobacteria (e.g. skin tests, lymphocyte transformation tests, and immunodiffusion).

2. Preliminary studies have attempted to identify which species should be further investigated. (a) Investigations using skin test reagents have shown that attention might be directed to $M$. vaccae, $M$. nonchromogenicum, and just possibly $M$. marinum among the 22 mycobacterial species investigated. (b) Investigations using laboratory animals and employing both skin tests and lymphocyte transformation have indicated a relationship between $M$. leprae and $M$. vaccae in particular.

\section{Present investigation}

1. Seven mycobacterial strains ( 4 of $M$. vaccae and 3 of $M$. nonchromogenicum) have been selected for special study. Stable rough variants of each strain are being isolated for use and will be preserved by freeze drying for later reference. The exact cultural biochemical and antigenic characteristics of each organism are to be determined, and each organism will be used to immunize guinea pigs, mice and rabbits in parallel with animals immunized with $M$. leprae from human material. Specific reagen ts will be prepared from each strain and used for:

(1) Skin testing the guinea pigs and mice 6 weeks after immunization.

(2) Lymphocyte transformation on lymph nodes of mice killed at the same time that other mice are skin tested.

(3) Immunodiffusion analysis, to be carried out using antisera from the immunized rabbits. This material will also be made available for crossed immunoelectrophoresis.

It is hoped that this experiment will further select the best strains of $M$. vaccae and $M$. nonchromogenicum for field investigations of leprosy patients and their contacts (provisionally Malawi might be considered as a suitable country for this study).

2. Studies of vaccines prepared from selected mycobacterial strains are being carried out in mice infected with $M$. leprae via the foot pad route.

3. An investigation has just begun into the acquisition of delayed hypersensitivity to those 
environmental bacteria encountered by experimental animals. This will be followed by attempts to suppress peripheral immunological responses by overloading with specific mycobacteria. The principal purpose of this experiment would be to produce an animal model for the apparent suppression of reactivity to leprosy bacilli seen in close contacts of the disease.

\section{Future studies}

1. Antigens prepared from organisms selected in the present studies will be included, together with those currently used, in the lymphocyte transformation studies on contacts of leprosy patients in Ethopia.

2. Dependent upon the completed analysis of the Burmese results, very carefully planned studies should be performed on the populations of selected villages in Burma. These villages would be chosen on the basis of different prevalences, and as far as possible incidences, of leprosy (high, middle and low). The reagents to be used would include a leprosy antigen (as selected from Protocol No. 7/75) and antigens prepared from the 2 or 3 most promising environmental species. Lymphocyte transformation as well as skin testing should be considered for this study.

\section{Problems arising}

1. Some degree of protection from tuberculosis is afforded by contact with certain other mycobacterial species, and a similar situation may complicate the epidemiology of leprosy.

2. The particular species selected as being related to the leprosy bacillus are themselves difficult to classify precisely, and much confusion exists as to their precise identity.

\section{PROTOCOL NO. 11/75. SKIN TESTS IN RELATION TO TRIALS OF POTENTIAL VACCINES}

\section{Introduction}

One of the primary objectives of the IMMLEP programme is the development of an effective antileprosy vaccine (IMM/74.3). Important applications of skin test antigens will be to help assess the effectiveness of the vaccine.

\section{Assessment before vaccination}

The protective effect of an antileprosy vaccine is likely to be affected by the epidemiological characteristics of the areas to be tested. Pre-vaccination skin testing may be important in several respects. For example:

1.1. Skin testing should be used to establish the age at which natural conversion occurs, to allow the test population to be vaccinated before extensive conversion due to exposure to $M$. leprae has taken place.

1.2. The proportion of positive reactors to skin tests amongst contacts of lepromatous and tuberculoid patients may vary from one area to another and affect the outcome of vaccination. If this is found to be the case, several areas should be tested, to allow vaccination to be undertaken in 2 areas with contrasting patterns.

Requirements. In order to allow a skin test to be used in this respect, it is necessary that it is not sensitizing and has a high specificity for $M$. leprae.

1.3. A strong reaction to the skin test may prove usefully prognostic of an adverse reaction to vaccination.

\section{Assessment of efficacy of vaccination}

The skin test antigen would be used to compare the ability of potential vaccines to produce sensitivity to antigens of $M$. leprae both in non-endemic and endemic areas.

Requirements. (1) The skin test must be non-sensitizing. A sensitizing skin test would be unsatisfactory, unless it was possible to use in vitro tests such as LTT before vaccination; (2) the reaction to the antigen must be correlated with protective immunity, (a) by ensuring that the degree of sensitivity to the antigen corresponds to the position of a leprosy patient on the 
Ridley-Jopling scale, (b) prospective studies in high risk groups comparing sensitivity to antigen with development of leprosy may also be considered.

\section{Post-vaccination assessment}

3.1. Non-converters and converters to be compared in a vaccine trial for subsequent development of leprosy. Detailed measurement of reaction sizes will be needed to draw maximum information from the trial.

3.2. Measurements of conversion rates and distribution of reaction sizes among various categories of contacts (it will be necessary also to measure natural conversion rates) should be compared with incidence rates of leprosy.

3.3. Lepromin testing (Mitsuda reaction) of at least a proportion of the population should also follow vaccination as part of a prospective trial to determine whether a negative reaction is associated with development of lepromatous leprosy.

\section{Problems}

4.1. The protocol is concerned with skin testing in relationship to vaccination. It will have to be modified as protocols for vaccination trials are developed.

4.2. It is possible that no skin test can be developed that will show complete correlation between reactivity and protective immunity. Nevertheless, skin testings may be useful to check potency of a vaccine and coverage of the population in a vaccination programme.

It should be borne in mind that lepromin (3.3. above) may interact with the vaccine to alter the level of protective immunity achieved. Lepromin testing may have to be confined to a proportion of the population. 